I N S T I T U T O

DE

M E D I C I N A

T R O P I C A L

DE

S ÃO PAULO

JOURNAL OF THE SÃO PAULO INSTITUTE OF TROPICAL MEDICINE

(1) University of Belgrade, School of

Medicine, Belgrade, Serbia

(2) Clinical Centre of Serbia, Clinic for

Pulmonology, Belgrade, Serbia

(3) Clinical Centre of Serbia, Clinic for Infectious and Tropic Diseases, Belgrade, Serbia

(4) University of Belgrade, School of Medicine, Institute for Pathology, Belgrade, Serbia

(5) Serbian Academy of Art and Science, Belgrade, Serbia

Correspondence to: Tatjana Adzic

Vukicevic

Clinical Centre of Serbia, Clinic for

Pulmonology, Koste Todorovica 2, 11000,

Belgrade, Serbia

E-mail: adzic_tatjana@yahoo.com

Received: 6 November 2016

Accepted: 22 February 2017
http://dx.doi.org/10.1590/S1678-9946201759020

\section{First reported case of fulminant TB with progression of infection from lungs to the genitourinary region}

\author{
Tatjana Adzic-Vukicevic ${ }^{1,2}$, Aleksandra Barac ${ }^{3}$, Aleksandra Dudvarski Ilic ${ }^{1,2}$ \\ Radmila Jankovic ${ }^{4}$, Jovan Hadzi-Djokic ${ }^{5}$, Dragica Pesut ${ }^{1,2}$
}

\section{ABSTRACT}

Although tuberculosis (TB) is a curable disease, it continues to be one of the leading infections associated with death in the world. Extra-pulmonary TB (EPTB) occurs in approximately $10 \%$ of the total cases, presenting with lymph nodes, pleura, bone and genitourinary tract as the most common locations. Genitourinary tuberculosis, the second most common EPTB, is very difficult to diagnose unless there is a high index of suspicion. Isolated TB orchitis or prostatitis without clinical evidence of renal involvement is a rare entity among genitourinary tuberculosis. We presented the first reported case of TB prostatitis and orchitis associated with pulmonary TB and the presence of an acute massive caseous pneumonia in an immunocompetent man. Despite the anti-TB therapy, the patient presented a rapid progression of disease and deterioration of general conditions taking to death, which occurred four days after TB treatment had started. Disseminated TB is a relatively uncommon cause of acute massive caseous pneumonia; however, there should always be suspicion of the disease, since it is a potentially treatable cause. This rare case supports the assertion that TB should be considered as an important differential diagnosis of genitourinary tumors irrespective of evidence of active TB elsewhere in the body.

KEYWORDS: Pulmonary tuberculosis. Caseous pneumonia. Tuberculous prostatitis. Orchitis. Immunocompetence

\section{INTRODUCTION}

Although tuberculosis (TB) is a curable disease, it continues to be one of the leading infections associated with death in the world ${ }^{1}$. Previous reports found that one third of the world's population is infected by Mycobacterium tuberculosis, and a very small inoculum is required for infection ${ }^{1,2}$. Serbia is a country with an intermediate to low TB incidence rate (18/ 100.000 inhabitants), low-middle HIV prevalence and has a $100 \%$ coverage of anti-TB treatment and vaccination at birth ${ }^{3}$. Miliary TB is a life-threatening disease resulting from the haematogenous spread of M. tuberculosis, and the lungs as the most commonly affected organ, although involvement of any organ is possible. Extra-pulmonary TB (EPTB) occurs in approximately $10 \%$ of the total cases with lymph node, bone and genitourinary tract as the most common locations ${ }^{4,5}$. We report the first case of tuberculous prostatitis and orchitis that resulted from the dissemination of a pulmonary TB. This immunocompetent man presented an acute massive caseous pneumonia.

\section{CASE REPORT}

A 55-years old man was admitted to the hospital due to a prolonged fever 
of up to $40^{\circ} \mathrm{C}$, cough and progressive dyspnoea that appeared 20 days before admission. His medical history included chronic obstructive pulmonary disease (COPD) and alcoholism. Physical examination revealed an afebrile, cachectic, adynamic and dyspnoeic patient with $5 \times 2 \mathrm{~cm}$ non-tender, bilateral scrotal masses. Chest X-ray showed bilateral miliary and larger non-homogenous as well as homogenous opacities with massive and excavated lesions on the left side (Figure 1A). Laboratory findings revealed elevated sedimentation rate $(42 / \mathrm{h})$; C-reactive protein (CRP) $162.6 \mathrm{mg} / \mathrm{L}$ (normal range: 0-8); normocytic anaemia with low haemoglobin level $110 \mathrm{~g} / \mathrm{L}$ (normal: 138-175); low total protein 51g/ L (normal: 62-81); low albumin level $18 \mathrm{~g} / \mathrm{L}$ (normal: 34-35); elevated amino transferases (AST) $150 \mathrm{U} / \mathrm{L}$ (normal: 0-37); alkaline transferases (ALT) 65U/ L (normal: 0-41); direct bilirubin $5 \mu \mathrm{mol} / \mathrm{L}$ (normal: 0-3.4); alkaline phosphatase (AF) $182 \mathrm{U} / \mathrm{L}$ (normal: 40-120); lactate dehydrogenase $854 \mathrm{U} / \mathrm{L}$ (normal: 220-460), and negative HIV-PCR test; HBsAg and anti-HCV antibodies. The electrocardiogram showed a sinus tachycardia with a heart rate of $100 / \mathrm{min}$. Arterial blood gas analysis showed an acute respiratory failure with $\mathrm{pO}_{2} 35.5, \mathrm{pCO}_{2} 25.8$ and saturation of $76 \%$. Sputum samples stained by Ziehl-Neelsen revealed the presence of acid-fast bacilli (AFB), while the tuberculin skin test was negative. A scrotal ultrasound was performed showing that the previous solid heterogenous mass had become a focal anechoic one with calcifications, which was compatible with a chronic inflammatory process. An exploration of both testis was performed through an inguinal incision. Approximately $30 \mathrm{~mL}$ of a purulent secretion containing caseous necrotic tissues was observed during the procedure. Frozen sections of the necrotic tissues were examined and revealed the presence of chronic granulomatous inflammation. The testis were then preserved, and the purulent secretion was sent for culture and Ziehl-Neelsen staining. The remaining necrotic tissues were sent for histopathological examination. The patient's urine was negative for TB culture and Ziehl-Neelsen staining. Nevertheless, histopathological examination of the necrotic tissues showed a caseating granulomatous reaction with Langhan's giant cells, and positive Ziehl-
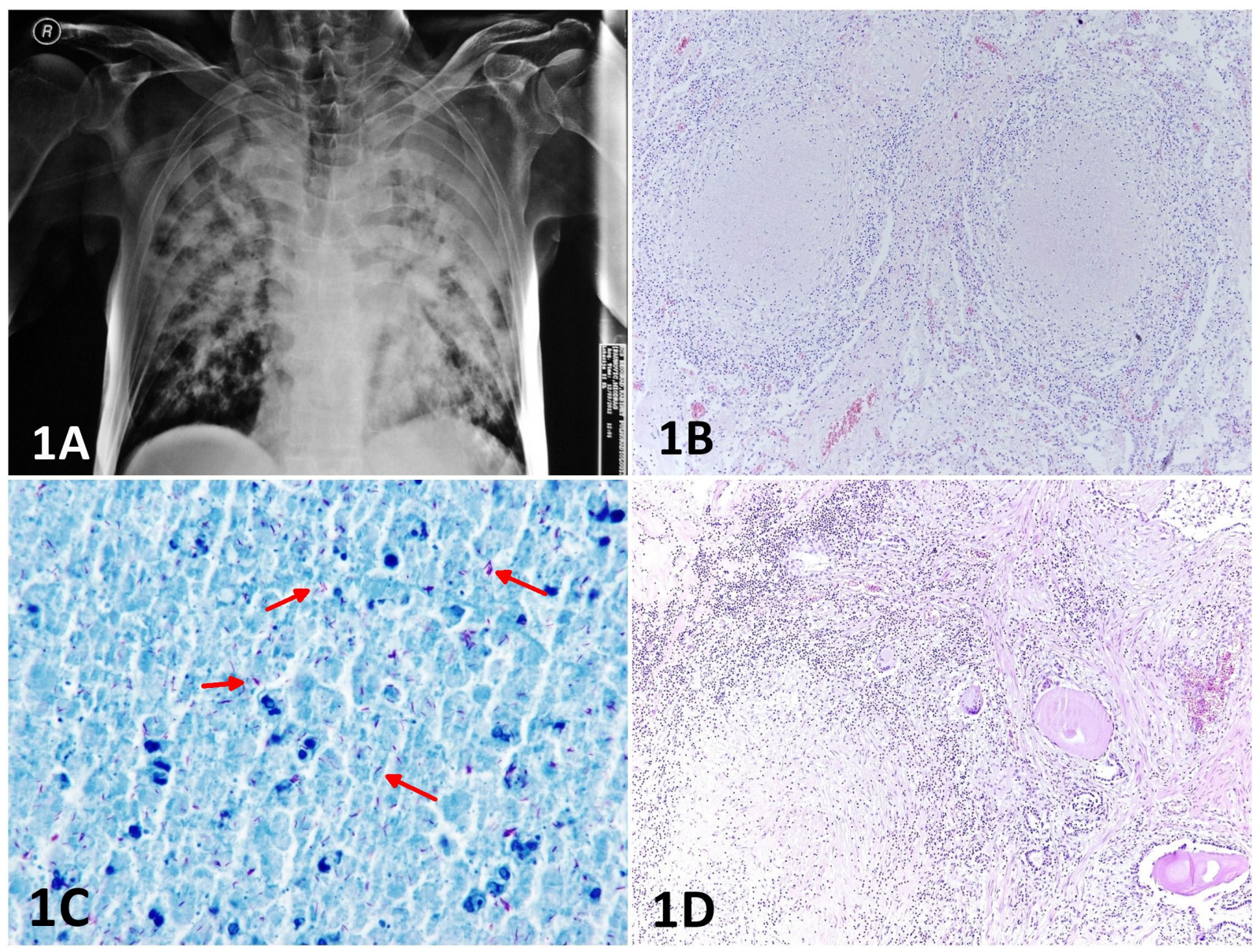

Figure 1 - 1A) Chest X-ray showing bilateral non-homogenous as well as homogenous opacities with excavated lesions; 1B) Miliary lung tuberculosis with tuberculous granulomas (H\&E x100); 1C) Caseosal necrosis in lungs containing acid fast bacilli, (Ziehl-Neelsen staining x100); 1D) Prostate with tuberculous granulomas (H\&E x100). 
Neelsen-stained bacilli. Treatment started with standard anti-TB drug regimen (isoniazid $300 \mathrm{mg} /$ day, rifampicin $600 \mathrm{mg} /$ day, pyrazinamide $1,200 \mathrm{mg} /$ day and ethambutol $1,200 \mathrm{mg} /$ day), with cefuroxime $4 \mathrm{~g} /$ day, continuous supply of oxygen through a nasopharyngeal tube with $4 \mathrm{~L} / \mathrm{min}$., saline and glycose solutions and albumin replacement. Although anti-TB, rehydration and symptomatic therapy was introduced, the patient died four days later. Autopsy revealed a disseminated miliary $\mathrm{TB}$ with a massive caseous pneumonia on the left lung, aside from a necrotic granulomatous orchitis and prostatitis with Langhans' giant cells. Histopathology and microbiology of the autopsied tissues revealed the presence of M. tuberculosis (Figure 1B, 1C and 1D).

The patient signed an informed consent agreeing with the publication of this case. Ethical approval was not required for this case report.

\section{DISCUSSION}

The present incidence and prevalence of TB has not changed significantly for decades due to the emigration of people born in endemic areas of TB, the growing population that are seropositive for the human immunodeficiency virus (HIV), and the emergence of multidrug resistant strains of Mycobacterium $^{6}$. EPTB can develop in a variety of locations, with the skeletal, genitourinary tract, and central nervous system as the three most common extrapulmonary sites $^{7}$. Evidences from the literature suggest that the infecting Mycobacterium tuberculosis bacilli reach the kidney through haematogenous spread from the lungs, then spread down to the ureter, bladder and/ or the prostate ${ }^{8}$. Progression of TB from pulmonary to genitourinary infection without clinical and laboratory evidence of renal involvement is very rare and difficult to diagnose ${ }^{9}$. Herein, we described the first case of fulminant TB with progression from pulmonary to genitourinary infection. Genitourinary TB is a form of EPTB that compromises the kidneys, ureters, testis and vas deferens $s^{1,2,4}$. Isolated prostate and epididymis involvement is unusual, and differential diagnosis includes neoplasia, infarction, acute and granulomatous infections ${ }^{2}$. Urine culture can yield false-negative results to Mycobacterium tuberculosis due to its low sensitivity (less than $50 \%)^{10}$. Ross et al. reported that renal TB or positive urine culture could be present during the later course of the disease ${ }^{11}$. Genitourinary TB is usually seen in young adults. In a review of 40 patients, Viswaroop et $a l .{ }^{9}$ reported that the median age was 32 years (range 21-37 years); our patient does not belong to this age category. Clinically, TB orchitis and prostatitis usually present with a painful scrotal swelling; however, it can be also a painless scrotal mass, being the first manifestation of TB infection of the prostate and seminal vesicles ${ }^{7}$. Voiding symptoms commonly seen in the acute inflammation of the epididymis and testis are not common in these patients ${ }^{9,12}$, as was noted in the reported case. Up to now, genitourinary TB has been reported presenting with the same imaging findings as those of other chronic inflammatory processes or testicular tumors. Commonly used imaging modalities such as scrotal ultrasonography, computed tomography scan, or magnetic resonance imaging may show diffuse or focal heterogenous lesions in the enlarged epididymis, with or without hydrocele, septation, extratesticular calcification, scrotal abscess, or scrotal sinus tract $^{9,12}$. A definitive diagnosis is based on hitopathological examination of material obtained by fine-needle aspiration for cytology or surgical resection ${ }^{4-7}$. However, fine needle aspiration is contraindicated in patients presenting with a painless scrotal tumor, because of possible lymphatic spread of malignant cells that may occur during aspiration for cytology, if the malignancy is present. Our patient had pulmonary TB with acute massive caseous pneumonia and secondary involvement of prostate and epididymis. Chest $\mathrm{X}$-ray indicated both, caseous pneumonia and miliary TB, while microbiological examination of sputum revealed AFB. Autopsy showed that military TB was disseminated from lungs to the genitourinary region, and was complicated by an acute massive caseous pneumonia that led to the lethal outcome. TB was formerly the main cause of death due to respiratory insufficiency ${ }^{4}$. There are two reasonable explanations for development of respiratory failure in our patient. The first reason was the infection caused by M. tuberculosis that produces a concentration of inflammatory cells within alveolar spaces, releasing granular enzymes and oxidants which participate in the alveolar membrane damage and lead to respiratory failure ${ }^{2,3}$. The second reason for the development of respiratory insufficiency in our patient was the hypoproteinaemia which has accelerated fluid exudation, promoting alveolar oedema, and contributing to the ventilation-perfusion imbalance, although, severe respiratory failure, adult respiratory distress syndrome (ARDS) should not be considered because of the adequate oxygenation provided by the nasopharyngeal tube ${ }^{5}$. Mortality due to respiratory failure caused by pulmonary TB has been reported to be over $60 \%$,

In our patient, liver function abnormalities were detected. This is compatible with miliary TB, as elevated $\mathrm{AF}$ and ALT were reported in $83 \%$ and $42 \%$ (respectively) of patients with $\mathrm{TB}^{2}$. Autopsy did not reveal granulomatous hepatitis. As it has been shown previously, the nutritional status defined by serum albumin concentration is an important predictor of survival ${ }^{3}$. Low serum albumin 
level in our patient indicated a poor prognosis that led to a lethal outcome. Presence of other comorbidities are also associated with higher mortality rates ${ }^{4}$. In the present case, presence of COPD and previous history of alcohol abuse were important risk factors for poor outcome.

TB of the genitourinary tract usually presents with atypical manifestations. Only $20 \%$ to $30 \%$ of patients with genitourinary TB have history of pulmonary infections. TB often affects the lower genitourinary system rather than the kidney and testis, bladder, ureter, prostate and penis as the most common affected sites in $\mathrm{men}^{2,5}$. Urine analysis may reveal sterile pyuria, haematuria or albuminuria. Diagnostic procedures for TB of the lower urinary tract include detection of $M$. tuberculosis in urine cultures or histopathological findings in tissues or positive polymerase chain reaction results in urine or tissues ${ }^{3,4}$. Tuberculin skin test was negative in our patient, and this was expected since the test is known to be negative in the most serious forms of the disease. The test is found to be positive only in $28-53 \%$ of patients with miliary $\mathrm{TB}$, so that a negative test result could not be used for TB exclusion ${ }^{5}$.

\section{CONCLUSION}

Disseminated TB which can be clinically missed, is usually diagnosed only post mortem. Disseminated TB is a relatively uncommon cause of acute massive caseous pneumonia; however, it should always be considered as it is a potentially treatable disease. Despite the rapid development of new anti-TB drugs in last few decades, mortality remains high, and treatment should be initiated as soon as the diagnosis is suspected. We presented the first case of TB prostatitis and orchitis disseminated from a pulmonary TB with acute massive caseous pneumonia in an immunocompetent man. It is difficult to achieve an early and correct diagnosis, which sometimes leads to an unnecessary orchiectomy. A high index of suspicion cannot be misregarded. Genitourinary TB is usually curable with anti-TB medication, while surgical resection is reserved for patients who do not respond to treatment. We recommend a scrotal ultrasound for patients when the initial diagnosis of painless scrotal tumor has become questionable.

\section{ETHICAL APPROVAL}

Ethical approval was not required for the study. The patient signed an informed consent for the publication of this case.

\section{CONFLICT OF INTERESTS}

The authors declare that there is no financial or other competing interest.

\section{REFERENCES}

1. Suthar AB, Zachariah R, Harries AD. Ending tuberculosis by 2030: can we do it? Int J Tuberc Lung Dis. 2016;20:1148-54.

2. Kim JY, Park YB, Kim YS, Kang SB, Shin JW, Park IW, et al. Miliary tuberculosis and acute respiratory distress syndrome. Int J Tuberc Lung Dis. 2003; 7:359-64.

3. Pesut DP, Bulajic MV, Lesic AR. Time trend and clinical pattern of extrapulmonary tuberculosis in Serbia, 1993-2007. Vojnosanit Pregl. 2012;69:227-30.

4. Nagavci BL, de Gelder R, Martikainen P, Deboosere P, Bopp M, Rychtaříková $\mathrm{J}$, et al. Inequalities in tuberculosis mortality: long-term trends in 11 European countries. Int J Tuberc Lung Dis. 2016;20:574-81.

5. Kho VK, Chan PH. Isolated tuberculous epididymitis presenting as a painless scrotal tumour. J Chin Med Assoc. 2012;75:292-5.

6. Yung-Ming C, Bao-Ying T, Yi-Chun W, Shih-Yan Y, Chang-Hsun C. Risk of Mycobacterium tuberculosis transmission in an aboriginal village, Taiwan. Southeast Asian J Trop Med Public Health 2006;37 Suppl 3:161-4.

7. Yu-Hung Lai A, Lu SH, Yu HJ, Kuo YC, Huang CY. Tuberculous epididymitis presenting as huge scrotal tumor. Urology. 2009;73:1163.e5-7.

8. Cinman AC. Genitourinary tuberculosis. Urology. 1982;20:353-8.

9. Viswaroop BS, Kekre N, Gopalakrishnan G. Isolated tuberculous epididymitis: a review of forty cases. J Postgrad Med. 2005;51:109-11.

10. Cousins DV, Wilton SD, Francis BR, Gow BL. Use of polymerase chain reaction for rapid diagnosis of tuberculosis. J Clin Microbiol. 1992;30:255-8.

11. Ross JC, Gow JG, St Hill CA. Tuberculous epididymitis: a review of 170 patients. Br J Sur. 1961;48:663-6.

12. Liu HY, Fu YT, Wu CJ, Sun GH. Tuberculous epididymitis: a case report and literature review. Asian J Androl. 2005; 7:329-32. 